\title{
Social Construction of Aging
}

\author{
Dr. Rashmi Saxena ${ }^{1 *}$
}

\section{ABSTRACT}

In life, we must take the good with the bad, and how we view these life events determines our well being and ability to adjust. Aging is not optional. We are all, in fact, aging from the moment we are born. The biggest issue regarding aging and getting old is how we look at it. Social constructivisms uncover the ways in which individuals participate in the creation of their perceived social reality. It involves looking at the ways old age are perceived, created, institutionalized, and made into traditions by human beings. Individuals construct their own life course through the choices and actions they take within the opportunities and constraints of history and social circumstances. This paper examines social construction of old age and aging in general and self-aging in particular among a small sample of 300 male and female respondents in the age range of $25-85$ years.

Main themes that emerged in the context constructions of general aging were as follows: good aging - 'all responsibilities being over'; worst part of growing old - 'empty nest'; females spend less time than males with elderly parents but expect a better relationship with them and are perceived to be the best support to elderly; best type of support for male elderly - 'emotional', for female elderly - 'physical'; best support that old parents can extend is in 'caring for children'; amount of advice that young adults are willing to accept from elderly - 'only a bit'; for old respondents caring for elderly - a 'government's responsibility.

When people were asked to construe their own old age they reported that the most predominant feeling in old age would be 'loneliness'; most important concern -'health'; expectations about living in old age - 'with son and their families'; possibilities of receiving day to day care from grown up children - 'great'.

Data analysis revealed several interesting findings by way of anxiety about aging, ageist attitudes and some utopian expectations. Most importantly, it indicated that even though people try to maintain a distance from elderly people, are unable to engage with them and/or care for their elderly parents they are sure of receiving, in their own old age, much better support, emotional closeness and daily care from their children.

\section{Keywords: Aging, Social Construction}

\footnotetext{
${ }^{1}$ Shri Ramswaroop Memorial Group of Professional College, Lucknow, India *Responding Author

(C) 2016, R Saxena; licensee IJIP. This is an Open Access Research distributed under the terms of the Creative Commons Attribution License (http://creativecommons.org/licenses/by/2.0), which permits unrestricted use, distribution, and reproduction in any Medium, provided the original work is properly cited.
} 


\section{Social Construction of Aging}

Aging refers to the processes of "accruing maturity with passage of time". Old age is the closing period in the life span. The goal of geriatrics is not to promote Senescence, but maximize the positive aspects of aging. In the words of the Gerontological Society of America, gerontology should be "adding life to years, not just more years to life".

Age generally refers to how old a person is, biologically, the biological process which brings about inevitable changes in outlook and behaviour, physical changes in the body, from teenage changes in hormones to elderly changes. However sociologists also point out that age is shaped by cultural norms and social factors, indifferent cultures age is viewed differently.

The roots of social construction can be found in sociology (Craig, 1995; Shotter and Gergen, 1994). Berger and Luckmann (1996) were first to define and comprehensively examine a phenomenon of social construction of reality. Social construction or social constructionism is a sociological theory of knowledge that considers how social phenomenon develops in particular social contexts. Within constructionist thought, a social construction is a concept or practice which may appear to be natural and obvious to those who accept it, but in reality is an invention or artifact of a particular culture or society. The implication is that social constructs are in some sense human choices rather than laws resulting from divine will or nature. Socially constructed reality is seen as an ongoing, dynamic process; reality is reproduced by people acting on their interpretation and their knowledge of it.

"Social construction is a cognitive and symbolic constructs that help individuals develop a sense of self, a sense of identity that is constructed in the process of interacting with others within a given human community".

By 'social construction' sociologists refer to the way that labels or classification are not naturally occurring but they are categories that we create. Saying the age is socially constructed suggests that a person's chronological age is affected by societies norms attached to age groups which in turn means that each group is expected to behave a certain way and to have a certain role.

Old age as a life stage is a socially constructed phenomenon though it is determined biologically as it is pointing out people who are 65 and over. A socially constructed world is not only performed by actors who are actively constructing their own worlds and but also by those who have influence upon those worlds through other social interventions. Social agents construct their own identities through the identity of others; hence in this case old age is constructed through being young; youth also construct the knowledge of old age.

Old age has "not only changed over time but has also varied among different cultures" (Hareven, 2005: 119). This is the strongest indicator which explains why old age should be seen as a reality

(C) The International Journal of Indian Psychology, ISSN 2348-5396 (e)| ISSN: 2349-3429 (p) | 134 


\section{Social Construction of Aging}

which is socially constructed instead of a natural phenomenon. According to Berger and Luckman (1967), reality, in general, is constructed through three stages; externalization, objectification and internalization. The child internalizes the world of his own significant others in the way his parents form it (Berger and Luckman, 1967:154). That is why the family in which one is born has important effects upon how he conceives of ageing.

\section{METHOD}

\section{Sample}

A purposive sample of 300 respondents living in lucknow was used for the present study. Half of these were males, the other half, females. Inclusion criteria consisted of having completed education till at least graduation, not being diagnosed with any illness at the time of the study and belonging to the middle socioeconomic status. The male and the female respondents were further subdivided into four age groups of 25-35 years, 45-65 years, and 65 years and above. In male sample $75 \%$ were graduate, $50 \%$ were post graduate and $25 \%$ were doing some professional courses whereas in female sample 95\% were graduate, $45 \%$ were postgraduate and $10 \%$ were doing some professional courses. All of them were married.

\section{Variables and Measures}

Two sets of variables were used in the present study. The first set consisted of the classificatory variables of sex (males vs. females) and age (25-35 years, 45-65 years and 65+ years). These two variables together led to a division of the sample into six subgroups of 'young', 'middle-aged', and 'old' males and females.

To set of questionnaire were administered to elicit respondents' narratives to explore their constructions regarding various aspects of old age. Some open ended single item measures were used to draw out narratives about general attitude toward old age (i.e., aging in general) and one's own aging in particular i.e., respondents were requested to imagine their own selves some 20-30 years from now.

\section{Procedure}

The questionnaires were constructed consisting of a demographic data sheet to measure the social construction of old age. The questionnaire was divided into two parts. The first part of the questionnaire contain seven single items measure which were used to elicit respondents orientation towards aging in general, the other part of the questionnaire contain six single items measure, regarding how people construe their old age i.e., aging in particular. A Hindi version of these questionnaires was prepared using the back translation method. Data were collected by administering the questionnaire in face-to-face personal interviews with the respondents. Respondents' narratives were then content analyzed. 


\section{RESULTS}

\section{Social construction of old age in general}

In this part a few open ended single item measures were used to explore respondent's narratives regarding attitude toward old age or aging through social construction among adult's respondents. To measure 'aging in general' respondents were asked to identify good aging, worst part of old age, support and help older people received, caring of older people these single item measures underlying people's construction of the aging process in general.

\section{(i) Factors which contribute to “aging well”}

Respondents were asked to prepare a list of factors which contribute to aging well. The important factors that contribute to graceful aging are an absence of physical health problems, diseases and financial problems, all responsibilities being over and having children spend time with their older parents. Both males and females in the middle age group listed "all responsibilities being over” as an important contributing factor for aging well.

Table 1: Factors contribute to aging well

\begin{tabular}{|c|c|c|c|c|c|c|c|c|c|c|}
\hline & & \multicolumn{4}{|l|}{ Males } & \multicolumn{4}{|c|}{ Females } & \multirow[b]{2}{*}{9} \\
\hline & & 1 & 2 & 3 & 4 & 5 & 6 & 7 & 8 & \\
\hline Z & 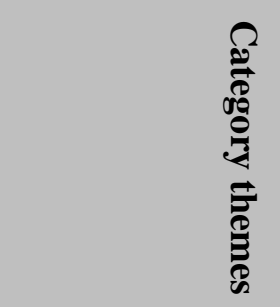 & 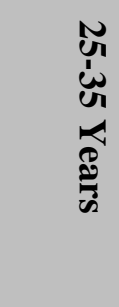 & 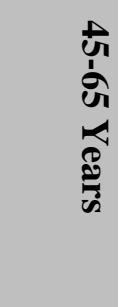 & 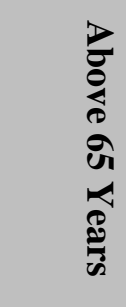 & 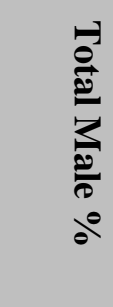 & 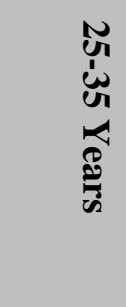 & 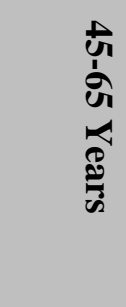 & 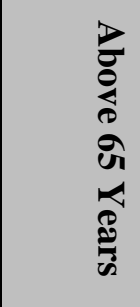 & 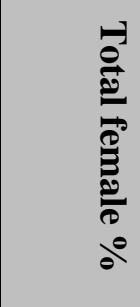 & $\frac{0}{0}$ \\
\hline 1 & $\begin{array}{l}\text { No physical } \\
\text { problem }\end{array}$ & 70.00 & 74.00 & 70.00 & 71.33 & 64.00 & 60.00 & 66.00 & 63.33 & 67.33 \\
\hline 2 & No diseases & 50.00 & 60.00 & 70.00 & 60.00 & 84.00 & 88.00 & 76.00 & 82.66 & 71.33 \\
\hline 3 & $\begin{array}{l}\text { No financial } \\
\text { problems }\end{array}$ & 92.00 & 84.00 & 76.00 & 84.00 & 94.00 & 84.00 & 82.00 & 86.66 & 85.33 \\
\hline 4 & $\begin{array}{l}\text { All } \\
\text { responsibilities } \\
\text { over }\end{array}$ & 94.00 & 96.00 & 86.00 & 92.00 & 80.00 & 90.00 & 88.00 & 86.00 & 89.00 \\
\hline 5 & $\begin{array}{l}\text { Children spend } \\
\text { time with their } \\
\text { old parents }\end{array}$ & 80.00 & 78.00 & 96.00 & 84.66 & 62.00 & 74.00 & 92.00 & 76.00 & 80.33 \\
\hline
\end{tabular}

\section{(ii) Worst part of growing old}

Loneliness, decline in physical health, empty nest syndrome, a lack of mobility and health related ignorance are the factors which are considered the worst aspects of old age. Old aged respondents reported that empty nest is the worst part of growing old.

(C) The International Journal of Indian Psychology, ISSN 2348-5396 (e) | ISSN: 2349-3429 (p) | 136 
Social Construction of Aging

\begin{tabular}{|c|c|c|c|c|c|c|c|c|c|c|}
\hline & & Males & & & & Femal & & & & \\
\hline & & 1 & 2 & 3 & 4 & 5 & 6 & 7 & 8 & 9 \\
\hline Z & 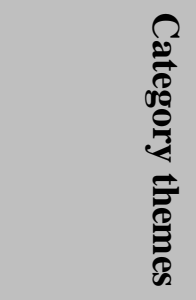 & 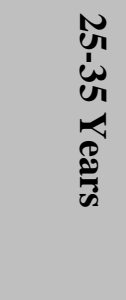 & 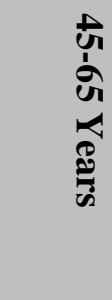 & 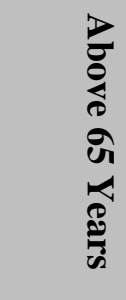 & 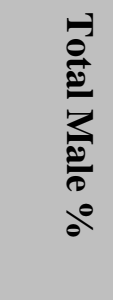 & 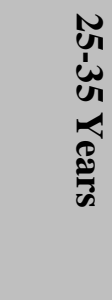 & 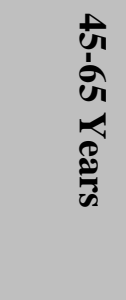 & 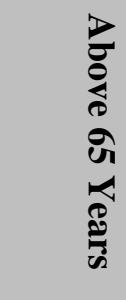 & 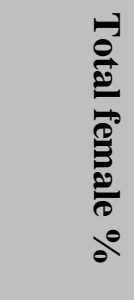 & $\frac{1}{0}$ \\
\hline 1 & Loneliness & 70.00 & 74.00 & 80.00 & 74.66 & 88.00 & 64.00 & 72.00 & 74.66 & 74.66 \\
\hline 2 & $\begin{array}{l}\text { Decline in } \\
\text { physical } \\
\text { strength }\end{array}$ & 64.00 & 76.00 & 66.00 & 68.66 & 64.00 & 60.00 & 76.00 & 66.66 & 67.66 \\
\hline 3 & Empty nest & 80.00 & 94.00 & 96.00 & 90.00 & 74.00 & 90.00 & 92.00 & 85.33 & 87.66 \\
\hline 4 & $\begin{array}{l}\text { Less } \\
\text { mobility }\end{array}$ & 40.00 & 50.00 & 60.00 & 50.00 & 58.00 & - & 30.00 & 29.33 & 39.66 \\
\hline 5 & Ignorance & 64.00 & 90.00 & 92.00 & 82.00 & 70.00 & 74.00 & 96.00 & 80.00 & 81.00 \\
\hline
\end{tabular}

(iii) Inclusion of old people in day to day life

For this a four point rating scale was developed which ranged from 1 - 'not at all' to 4 - 'too much'. The data analysis indicated that as compared to young and old respondent's middle aged males (age range 45-65 years) are perceived to involve their older parent more in the daily life.

\begin{tabular}{|c|c|c|c|c|c|c|c|c|c|c|}
\hline & & \multicolumn{4}{|l|}{ Males } & \multicolumn{4}{|c|}{ Females } & \multirow[b]{2}{*}{9} \\
\hline & & 1 & 2 & 3 & 4 & 5 & 6 & 7 & 8 & \\
\hline zz & 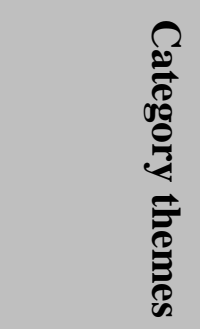 & 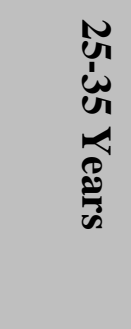 & 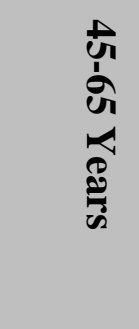 & 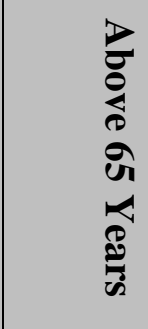 & 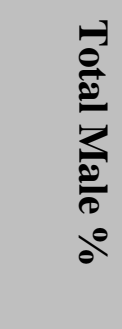 & 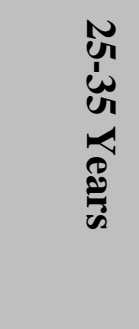 & 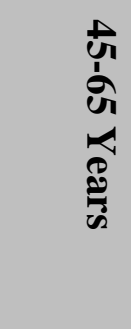 & 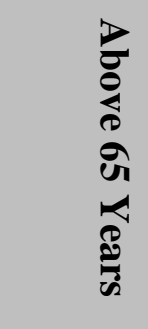 & 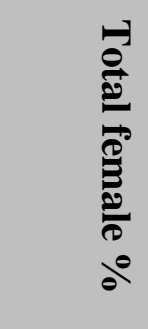 & مُ \\
\hline 1 & Not at all & - & - & - & - & - & - & - & - & - \\
\hline 2 & Little bit & 4.00 & & 80.00 & 42.00 & 50.00 & 56.00 & 90.00 & 65.33 & 56.00 \\
\hline 3 & A bit & 76.00 & 90.00 & 10.00 & 58.66 & 24.00 & 16.00 & 8.00 & 16.00 & 37.33 \\
\hline 4 & Too much & 20.00 & 10.00 & 10.00 & 13.33 & 26.00 & 6.00 & 2.00 & 11.33 & 12.33 \\
\hline
\end{tabular}

(iv) Support/help received from sons Vs. daughters

It is seen that the old males (above 65 years) reported that old people receive more help and support from their daughters than from their sons. Among females also daughters are perceived as more important caregivers in comparison to sons and other family members.

(C) The International Journal of Indian Psychology, ISSN 2348-5396 (e)| ISSN: 2349-3429 (p) | 137 


\section{Social Construction of Aging}

A comparison of all males vs. all females shows that females, as compared to males, more often identified daughters rather than sons as potential persons giving support and help to their older parents.

\begin{tabular}{|c|c|c|c|c|c|c|c|c|c|c|c|}
\hline & & Males & & & & Female & & & & & \\
\hline & & 1 & 2 & 3 & 4 & 5 & 6 & 7 & 8 & & 9 \\
\hline Z' & 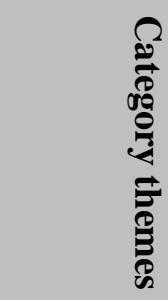 & 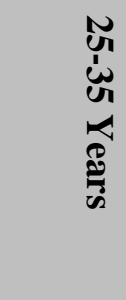 & 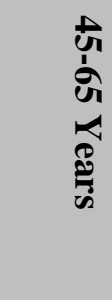 & 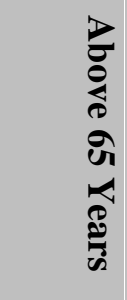 & 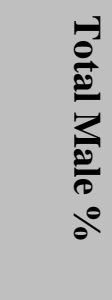 & 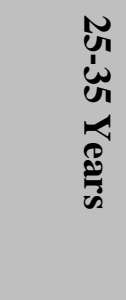 & 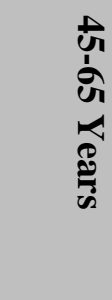 & 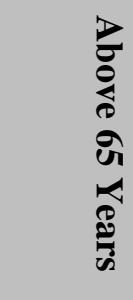 & & 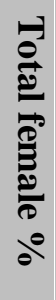 & $\frac{{ }^{0}}{\stackrel{0}{2}}$ \\
\hline 1 & Son & 80.00 & 50.00 & 30.00 & 53.33 & 50.00 & 60.00 & 44.00 & 51.33 & & 52.33 \\
\hline 2 & Daughter & 40.00 & 90.00 & 96.00 & 75.33 & 76.00 & 92.00 & 94.00 & 87.33 & & 81.33 \\
\hline 3 & $\begin{array}{l}\text { Other } \\
\text { family } \\
\text { members }\end{array}$ & 10.00 & 20.00 & 10.00 & 13.33 & 30.00 & 20.00 & 16.00 & 22.00 & & 17.66 \\
\hline
\end{tabular}

\section{(v) Help/Support expected by old parents}

The important areas where in old people are perceived to be useful are giving advice, caring for grand children, offering financial support, sharing house hold chores/ activities and providing guidance.

\begin{tabular}{|c|c|c|c|c|c|c|c|c|c|c|c|}
\hline & & \multicolumn{4}{|l|}{ Males } & \multicolumn{4}{|c|}{ Females } & \multirow{2}{*}{\multicolumn{2}{|c|}{9}} \\
\hline & & 1 & 2 & 3 & 4 & 5 & 6 & 7 & 8 & & \\
\hline Zu & 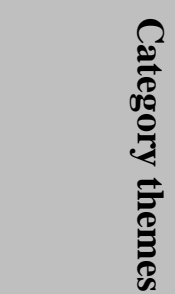 & 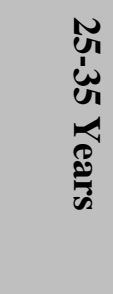 & 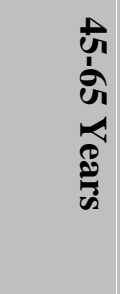 & 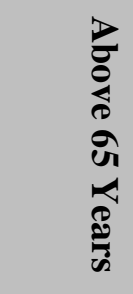 & 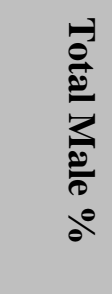 & 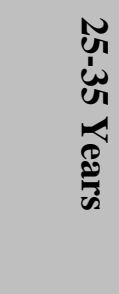 & 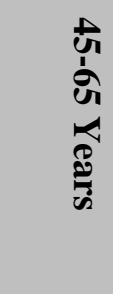 & 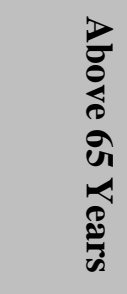 & 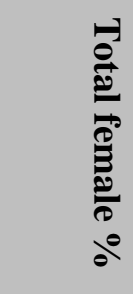 & & $\frac{{ }_{0}^{0}}{\stackrel{0}{0}}$ \\
\hline 1 & $\begin{array}{l}\text { By giving } \\
\text { them } \\
\text { advice }\end{array}$ & 50.00 & 60.00 & 70.00 & 60.00 & 84.00 & 88.00 & 76.00 & 82.66 & 71.33 & \\
\hline 2 & $\begin{array}{l}\text { Caring } \\
\text { Grand } \\
\text { children }\end{array}$ & 94.00 & 96.00 & 86.00 & 92.00 & 80.00 & 90.00 & 88.00 & 86.00 & 89.00 & \\
\hline 3 & $\begin{array}{l}\text { Financial } \\
\text { Support }\end{array}$ & 96.00 & 80.00 & 94.00 & 90.00 & 90.00 & 74.00 & 92.00 & 85.33 & 87.66 & \\
\hline
\end{tabular}

(C) The International Journal of Indian Psychology, ISSN 2348-5396 (e) | ISSN: 2349-3429 (p) | 138 
Social Construction of Aging

\begin{tabular}{|c|c|c|c|c|c|c|c|c|c|c|c|}
\hline & & \multicolumn{4}{|l|}{ Males } & \multicolumn{4}{|c|}{ Females } & \multirow{2}{*}{\multicolumn{2}{|c|}{9}} \\
\hline & & 1 & 2 & 3 & 4 & 5 & 6 & 7 & 8 & & \\
\hline Z & 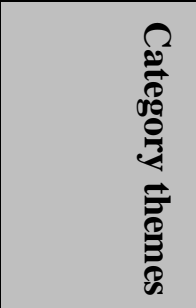 & 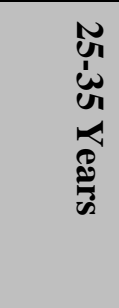 & 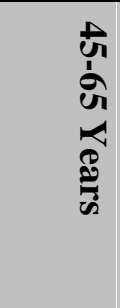 & 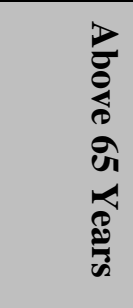 & 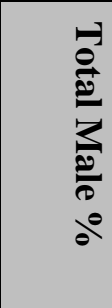 & 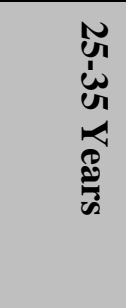 & 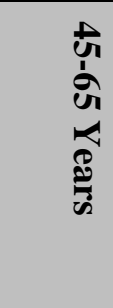 & 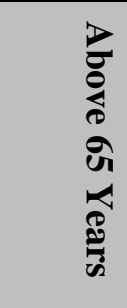 & 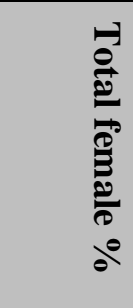 & & مْ \\
\hline 4 & $\begin{array}{l}\text { By doing } \\
\text { some } \\
\text { house } \\
\text { hold } \\
\text { activities }\end{array}$ & 92.00 & 84.00 & 76.00 & 84.00 & 94.00 & 84.00 & 82.00 & 86.66 & 85.33 & \\
\hline 5 & $\begin{array}{l}\text { By } \\
\text { providing } \\
\text { guidance }\end{array}$ & 70.00 & 74.00 & 70.00 & 71.33 & 64.00 & 60.00 & 66.00 & 63.33 & 67.33 & \\
\hline
\end{tabular}

(vi) Help or Support expected by young people

The important themes on this item correspond to financial support, caring for old people's needs, listening to them, spending time with old parents and providing physical and emotional support to them. Caring for older people needs is the most important support for older people that younger people give

\begin{tabular}{|c|c|c|c|c|c|c|c|c|c|c|c|}
\hline & & \multicolumn{4}{|l|}{ Males } & \multicolumn{4}{|c|}{ Females } & \multirow{2}{*}{\multicolumn{2}{|c|}{9}} \\
\hline & & 1 & 2 & 3 & 4 & 5 & 6 & 7 & 8 & & \\
\hline za & 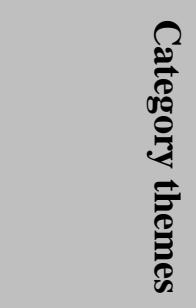 & 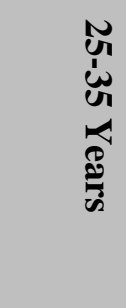 & 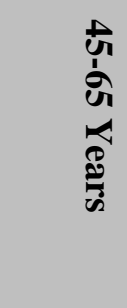 & 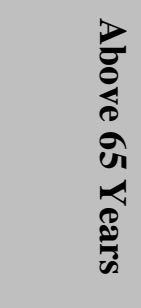 & 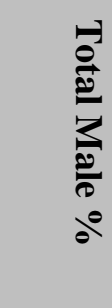 & 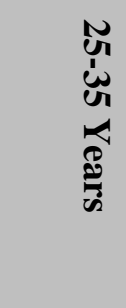 & 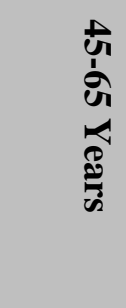 & 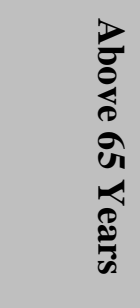 & 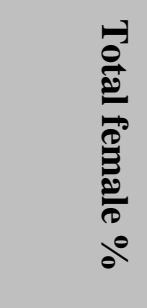 & व & \\
\hline 1 & $\begin{array}{l}\text { Financial } \\
\text { support }\end{array}$ & 60.00 & 50.00 & 40.00 & 50.00 & 58.00 & - & 30.00 & 29.33 & 39.66 & \\
\hline 2 & $\begin{array}{l}\text { By caring } \\
\text { old people } \\
\text { needs }\end{array}$ & 88.00 & 94.00 & 90.00 & 90.66 & 96.00 & 86.00 & 84.00 & 88.66 & 89.66 & \\
\hline 3 & $\begin{array}{l}\text { By } \\
\text { listening } \\
\text { them }\end{array}$ & 70.00 & 74.00 & 80.00 & 74.00 & 88.00 & 64.00 & 72.00 & 74.66 & 74.66 & \\
\hline
\end{tabular}

(c) The International Journal of Indian Psychology, ISSN 2348-5396 (e)| ISSN: 2349-3429 (p) | 139 
Social Construction of Aging

\begin{tabular}{|c|c|c|c|c|c|c|c|c|c|c|c|}
\hline & & \multicolumn{4}{|l|}{ Males } & \multicolumn{4}{|c|}{ Females } & \multirow{2}{*}{\multicolumn{2}{|c|}{9}} \\
\hline & & 1 & 2 & 3 & 4 & 5 & 6 & 7 & 8 & & \\
\hline Zב & 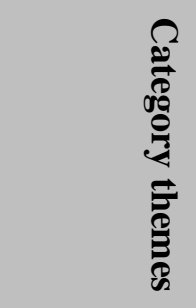 & 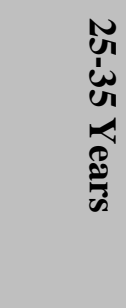 & 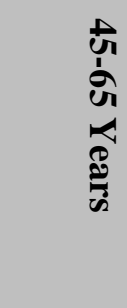 & 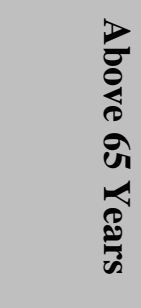 & 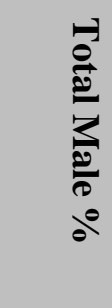 & 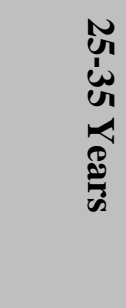 & 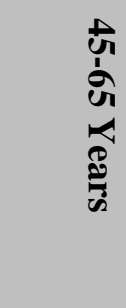 & 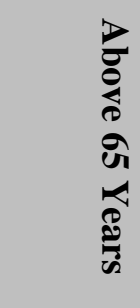 & 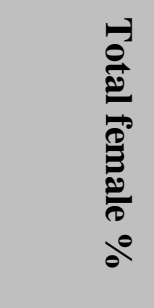 & & مُ \\
\hline 4 & $\begin{array}{l}\text { Spend } \\
\text { more time } \\
\text { with old } \\
\text { parents }\end{array}$ & 94.00 & 80.00 & 96.00 & 90.00 & 90.00 & 74.00 & 92.00 & 85.33 & 87.66 & \\
\hline 5 & $\begin{array}{l}\text { Physical } \\
\text { support }\end{array}$ & 64.00 & 76.00 & 66.00 & 68.66 & 64.00 & 60.00 & 76.00 & 66.66 & 67.66 & \\
\hline 6 & $\begin{array}{l}\text { Emotional } \\
\text { support }\end{array}$ & 92.00 & 90.00 & 64.00 & 82.00 & 96.00 & 74.00 & 70.00 & 80.00 & 81.00 & \\
\hline
\end{tabular}

\section{(vii) Old parents as a source of burden}

Adult respondents were asked to indicate the extent to which caring for old parents is a source of burden. For all age groups of male and females, caring for old parents was reported (at least verbally!) to be a 'no burden at all'.

\begin{tabular}{|c|c|c|c|c|c|c|c|c|c|c|c|}
\hline & & \multicolumn{4}{|l|}{ Males } & \multicolumn{4}{|c|}{ Females } & \multirow{2}{*}{\multicolumn{2}{|c|}{9}} \\
\hline & & 1 & 2 & 3 & 4 & 5 & 6 & 7 & 8 & & \\
\hline ?' & 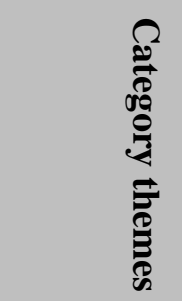 & 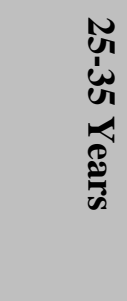 & 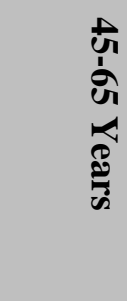 & 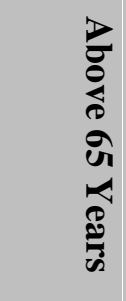 & 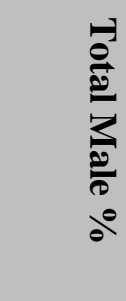 & 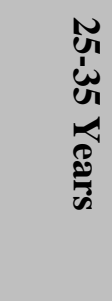 & 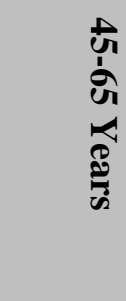 & 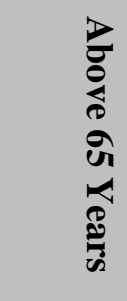 & 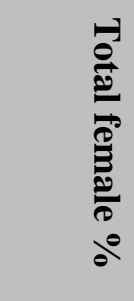 & & $\frac{\sqrt{0}}{\stackrel{0}{\Theta}}$ \\
\hline 1 & $\begin{array}{l}\text { No } \\
\text { burden at } \\
\text { all }\end{array}$ & 100.00 & 100.00 & 100.00 & 100.00 & 96.00 & 100.00 & 100.00 & 98.66 & 99.33 & \\
\hline 2 & $\begin{array}{l}\text { A little } \\
\text { burden }\end{array}$ & - & - & - & - & 4.00 & - & - & - & - & \\
\hline 3 & $\begin{array}{l}\text { Moderate } \\
\text { burden }\end{array}$ & - & - & - & - & - & - & - & - & - & \\
\hline 4 & $\begin{array}{l}\text { Lot of } \\
\text { burden }\end{array}$ & - & - & - & - & - & - & - & - & - & \\
\hline
\end{tabular}

(c) The International Journal of Indian Psychology, ISSN 2348-5396 (e)| ISSN: 2349-3429 (p) | 140 


\section{Social Construction of Aging}

(viii) Caring for old parents as a source of satisfaction

An attempt was next made to assess the extent to which caring for the older parents could be a source of satisfaction for their children. All males and females in various age groups reported that providing care for their older parents would lead to a lot of satisfaction for them.

\begin{tabular}{|c|c|c|c|c|c|c|c|c|c|c|}
\hline & & Males & & & & Femal & & & & \\
\hline & & 1 & 2 & 3 & 4 & 5 & 6 & 7 & 8 & 9 \\
\hline 'z & 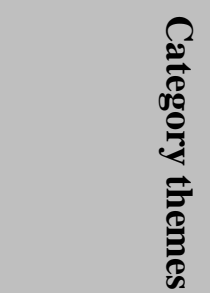 & 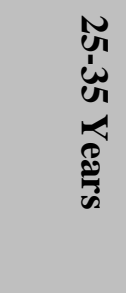 & 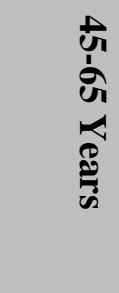 & 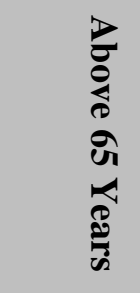 & 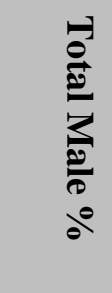 & 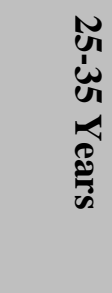 & 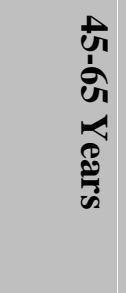 & 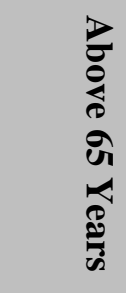 & 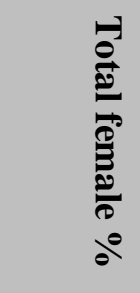 & 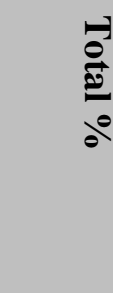 \\
\hline 1 & Not at all & - & - & - & - & - & - & - & - & - \\
\hline 2 & $\begin{array}{l}\text { A little } \\
\text { satisfaction }\end{array}$ & 20.00 & 10.00 & - & - & - & - & - & - & 5.00 \\
\hline 3 & $\begin{array}{l}\text { Moderate } \\
\text { satisfaction }\end{array}$ & 10.00 & 10.00 & - & & 6.00 & 4.00 & - & - & 7.5 \\
\hline 4 & $\begin{array}{l}\text { Lot of } \\
\text { satisfaction }\end{array}$ & 70.00 & 90.00 & 100.00 & - & 94.00 & 96.00 & 100.00 & - & 91.66 \\
\hline
\end{tabular}

(ix) Responsibility of caring for older people

As compared to older and younger generations, the middle aged males think that children are responsible for caring for their old parents. Many older males responded that government is responsible for caring for older people. Among females the middle aged ones more often thought that children are responsible for caring for their older people.

\begin{tabular}{|c|c|c|c|c|c|c|c|c|c|c|}
\hline & & \multicolumn{4}{|l|}{ Males } & \multicolumn{4}{|c|}{ Females } & \multirow[b]{2}{*}{9} \\
\hline & & 1 & 2 & 3 & 4 & 5 & 6 & 7 & 8 & \\
\hline Zz & 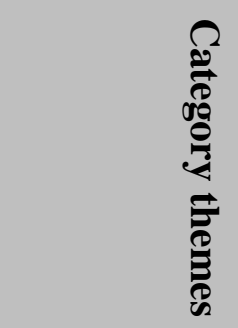 & 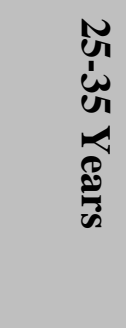 & 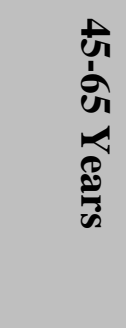 & 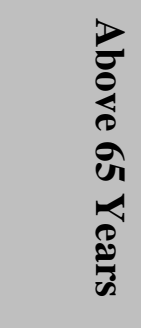 & 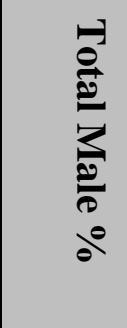 & 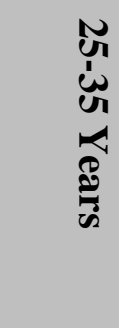 & 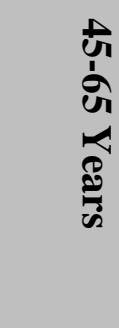 & 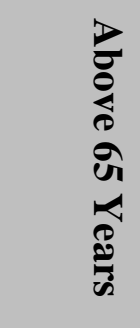 & 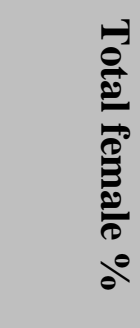 & $\frac{\stackrel{3}{0}}{\stackrel{0}{\varrho}}$ \\
\hline 1 & $\begin{array}{l}\text { Old people } \\
\text { themselves }\end{array}$ & 50.00 & 30.00 & 70.00 & 50.00 & 56.00 & 40.00 & 80.00 & 58.66 & 54.33 \\
\hline 2 & $\begin{array}{l}\text { Their } \\
\text { children }\end{array}$ & 70.00 & 90.00 & 50.00 & 70.00 & 90.00 & 92.00 & 40.00 & 74.00 & 72.00 \\
\hline 3 & Some NGO & 40.00 & 24.00 & 40.00 & 34.66 & 30.00 & 20.00 & 10.00 & 20.00 & 27.33 \\
\hline 4 & Government & 76.00 & 80.00 & 84.00 & 80.00 & 40.00 & 50.00 & 70.00 & 53.33 & 70.00 \\
\hline
\end{tabular}

(c) The International Journal of Indian Psychology, ISSN 2348-5396 (e)| ISSN: 2349-3429 (p) | 141 


\section{Social Construction of Aging}

\section{(x) Adults' responsibility towards their own children vs. parents}

Total sample responded that they consider themselves equally responsible for their children as well as their parents.

\begin{tabular}{|c|c|c|c|c|c|c|c|c|c|c|}
\hline & & \multicolumn{4}{|l|}{ Males } & \multicolumn{4}{|c|}{ Females } & \multirow[b]{2}{*}{9} \\
\hline & & 1 & 2 & 3 & 4 & 5 & 6 & 7 & 8 & \\
\hline ? & 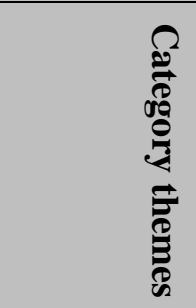 & 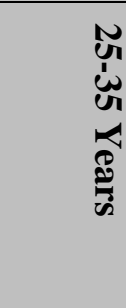 & 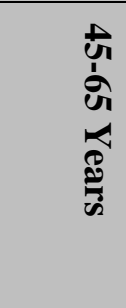 & 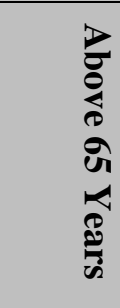 & 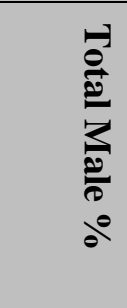 & 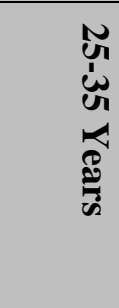 & 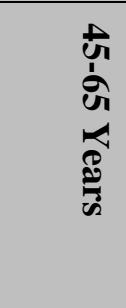 & 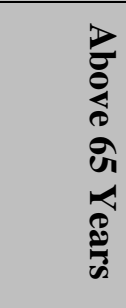 & 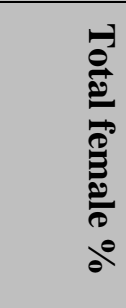 & $\frac{\overbrace{}^{-0}}{\stackrel{0}{0}}$ \\
\hline 1 & $\begin{array}{l}\text { Only } \\
\text { towards his } \\
\text { or her } \\
\text { children }\end{array}$ & - & - & - & - & - & - & - & - & - \\
\hline 2 & $\begin{array}{l}\text { Mostly for } \\
\text { children } \\
\text { and a little } \\
\text { towards } \\
\text { parents }\end{array}$ & - & - & - & - & - & - & - & - & - \\
\hline 3 & $\begin{array}{l}\text { Equally for } \\
\text { children as } \\
\text { well as } \\
\text { parents }\end{array}$ & 100.00 & 100.00 & 100.00 & 100.00 & 100.00 & 100.00 & 100.00 & 100.00 & 100.00 \\
\hline 4 & $\begin{array}{l}\text { Towards } \\
\text { parents } \\
\text { more than } \\
\text { children }\end{array}$ & - & - & - & - & - & - & - & - & - \\
\hline
\end{tabular}

(xi) Important problems of aging

Poor health is the major problem according to all sub-groups inclusive of males and females. Second major problem of old age as reported by the respondents is loneliness. 
Social Construction of Aging

\begin{tabular}{|c|c|c|c|c|c|c|c|c|c|c|}
\hline & & Males & & & & Femal & & & & \\
\hline & & 1 & 2 & 3 & 4 & 5 & 6 & 7 & 8 & 9 \\
\hline za & 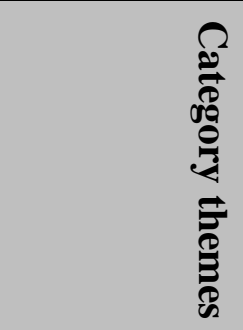 & 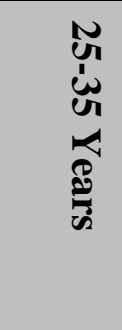 & 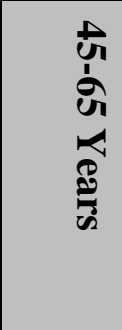 & 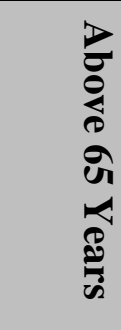 & 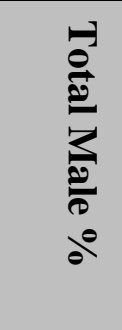 & 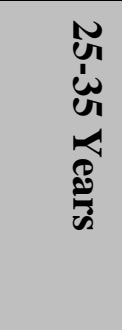 & 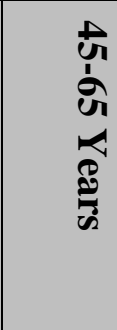 & 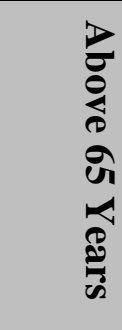 & 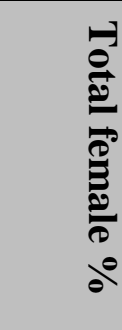 & $\frac{0}{0}$ \\
\hline 1 & $\begin{array}{l}\text { Not having } \\
\text { enough work } \\
\text { to keep } \\
\text { meaningfully } \\
\text { busy }\end{array}$ & 30.00 & - & 20.00 & 25.00 & 40.00 & 20.00 & 30.00 & 30.00 & 23.33 \\
\hline 2 & $\begin{array}{l}\text { Not having } \\
\text { enough to } \\
\text { live } \\
\text { comfortably }\end{array}$ & 20.00 & - & - & - & - & - & - & - & - \\
\hline 3 & Poor health & 70.00 & 90.00 & 96.00 & 85.33 & 88.00 & 94.00 & 96.00 & 92.66 & 89.00 \\
\hline 4 & Loneliness & 80.00 & 70.00 & 80.00 & 76.66 & 74.00 & 70.00 & 76.00 & 73.33 & 75.00 \\
\hline 5 & $\begin{array}{l}\text { Irritability \& } \\
\text { bad temper }\end{array}$ & 40.00 & 30.00 & 50.00 & 40.00 & 36.00 & 42.00 & 50.00 & 42.66 & 41.33 \\
\hline
\end{tabular}

\section{Social construction of one's own old age some 20-30 years from now}

To measure one's own aging in particular respondents were requested to imagine their own selves some 20-30 years from now. They were requested to close their eyes and transport themselves 20 years into the future and think of how they might feel, think, do, have problems, handle these problems, get support, type of support, be useful, spend time with their own old parents, receive advice from parents and relate with them.

\section{(i) Feelings in old age}

Respondents were next asked to indicate their feelings after 20-30 years. The important feelings in that situations emerged to be loneliness, irritation and isolation. 
Social Construction of Aging

\begin{tabular}{|c|c|c|c|c|c|c|c|c|c|c|}
\hline & & \multicolumn{4}{|c|}{ Males } & \multicolumn{4}{|c|}{ Females } & \multirow[b]{2}{*}{9} \\
\hline & & 1 & 2 & 3 & 4 & 5 & 6 & 7 & 8 & \\
\hline$\underbrace{}_{0}$ & 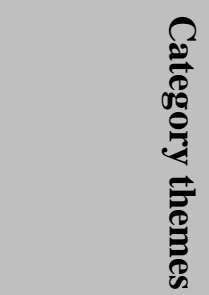 & 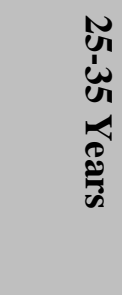 & 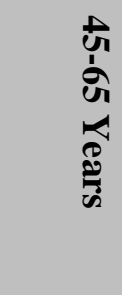 & 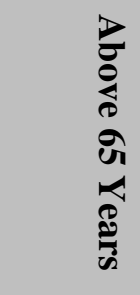 & 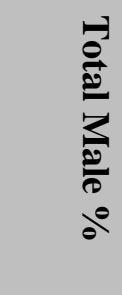 & 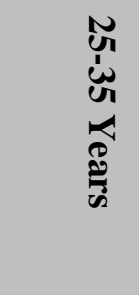 & 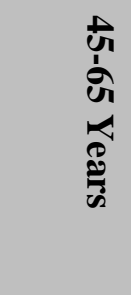 & 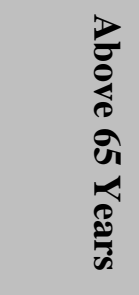 & 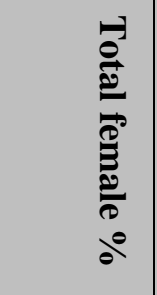 & $\frac{0}{0}$ \\
\hline 1 & Loneliness & 88.00 & 80.00 & 96.00 & 80.00 & 64.00 & 76.00 & 82.00 & 74.00 & 81.00 \\
\hline 2 & Irritability & 60.00 & 50.00 & 40.00 & 50.00 & 58.00 & - & 30.00 & 29.33 & 39.66 \\
\hline 3 & Isolation & 64.00 & 70.00 & 90.00 & 74.66 & 70.00 & 74.00 & 96.00 & 80.00 & 90.33 \\
\hline
\end{tabular}

(ii) Major future worries 20-30 years from now

Respondents were asked to prepare a list of worries which they may experience in future, some 20-30 years from now. The main worries of the respondents in old age are - loss of loved one, health concerns, dependency and financial concerns. Worry related to health is the most important for all males and females. After health, the next important worry is financial difficulty.

\section{(iii) Living with family members}

Participants were asked to visualize which family members they may be living with 20-30 years later. The highest percentages regarding this dimension are son and daughter-in law and the second is with their spouse.

\begin{tabular}{|c|c|c|c|c|c|c|c|c|c|c|c|}
\hline & & & Males & & & & & ales & & & \\
\hline & & 1 & 2 & 3 & 4 & 5 & 6 & & 7 & 8 & 9 \\
\hline${ }_{0}^{2}$ & 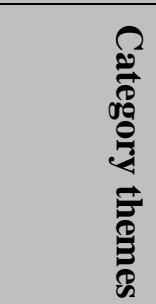 & 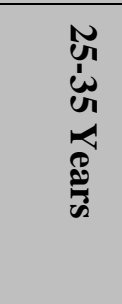 & 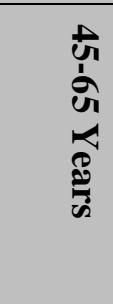 & 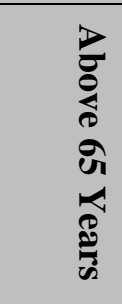 & 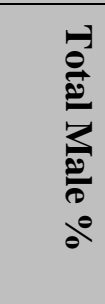 & 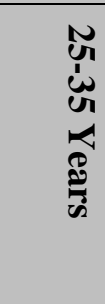 & & 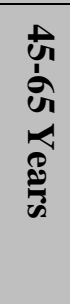 & 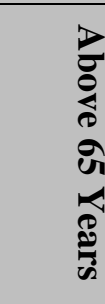 & 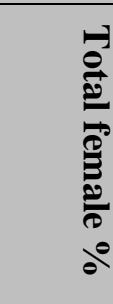 & $\frac{{ }^{3}}{0^{\circ}}$ \\
\hline 1 & $\begin{array}{l}\text { Son and } \\
\text { daughter } \\
\text { in law }\end{array}$ & 70.00 & 90.00 & 96.00 & 85.33 & 88.00 & 94.00 & & 96.00 & 92.66 & 89.00 \\
\hline 2 & Spouse & 80.00 & 94.00 & 96.00 & 90.00 & 74.00 & 90.00 & & 92.00 & 85.33 & 87.66 \\
\hline 3 & $\begin{array}{l}\text { Grand } \\
\text { Children }\end{array}$ & 76.00 & 84.00 & 92.00 & 84.00 & 82.00 & 84.00 & & 94.00 & 86.66 & 85.33 \\
\hline
\end{tabular}

(C) The International Journal of Indian Psychology, ISSN 2348-5396 (e)| ISSN: 2349-3429 (p) | 144 


\section{Social Construction of Aging}

\section{(iv) Future problems}

Important responses regarding this single item are physical problems, financial problems and emotional problems. Out of these three problems, the problems related to emotions is the most important one in old age for all males and females irrespective of their different age groups.

\begin{tabular}{|c|c|c|c|c|c|c|c|c|c|c|}
\hline & & & Males & & & & Females & & & \\
\hline & & 1 & 2 & 3 & 4 & 5 & 6 & 7 & 8 & 9 \\
\hline za & 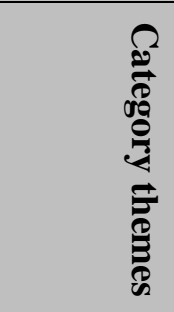 & 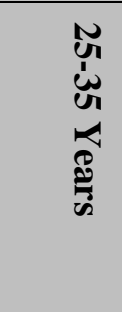 & 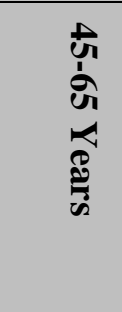 & 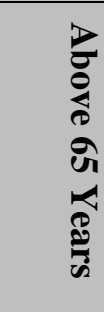 & 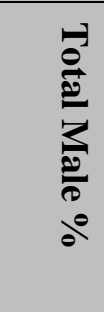 & 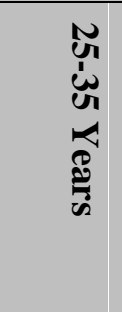 & 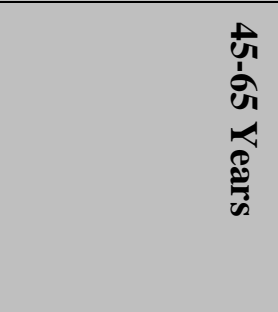 & 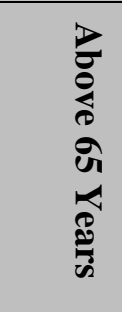 & 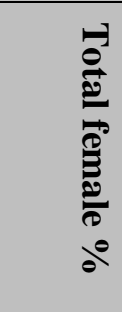 & 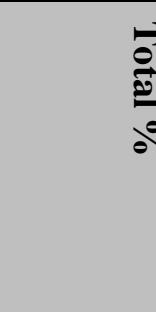 \\
\hline 1 & Financial & 60.00 & 80.00 & 96.00 & 78.66 & 70.00 & 88.00 & 80.00 & 79.33 & 79.00 \\
\hline 2 & Physical & 70.00 & 74.00 & 84.00 & 76.00 & 60.00 & 76.00 & 92.00 & 76.00 & 76.00 \\
\hline 3 & Emotional & 84.00 & 86.00 & 90.00 & 60.66 & 68.00 & 74.00 & 80.00 & 74.00 & 80.33 \\
\hline
\end{tabular}

\section{(v) Possibilities of getting support at that age}

Female respondents are clearly more hopeful than male respondents about getting the desired support from their young ones in future. It could be because females usually provide more care and support and hence they also anticipate receiving more care and support. Or else, females are less reality oriented than males.

\begin{tabular}{|c|c|c|c|c|c|c|c|c|c|c|}
\hline & & & Males & & & & Females & & & \\
\hline & & 1 & 2 & 3 & 4 & 5 & 6 & 7 & 8 & 9 \\
\hline Zz & 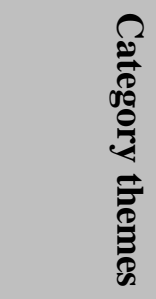 & 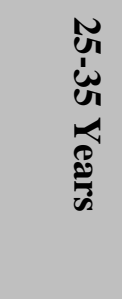 & 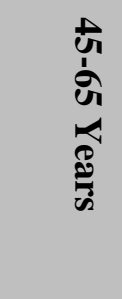 & 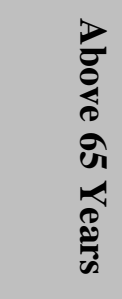 & 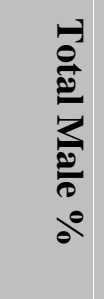 & 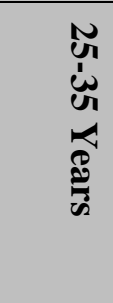 & 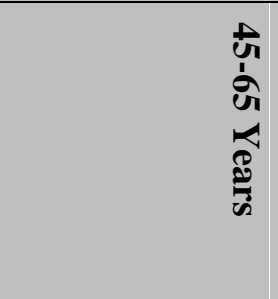 & 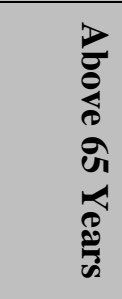 & 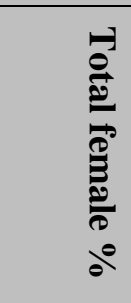 & 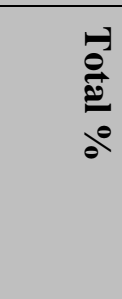 \\
\hline 1 & $0-10 \%$ & & & & & & & & & \\
\hline 2 & $20-30 \%$ & & & & & & & & & \\
\hline 3 & $40-50 \%$ & & & 86.00 & & & & & & \\
\hline 4 & $60-70 \%$ & 16.00 & 20.00 & 14.00 & & 8.00 & 30.00 & 12.00 & & \\
\hline 5 & $80-90 \%$ & 92.00 & 80.00 & & & 96.00 & 70.00 & 88.00 & & \\
\hline 6 & $100 \%$ & & & & & & & & & \\
\hline
\end{tabular}

\section{(vi) Type of support}

An attempt was next made to assess what types of support respondents would likely get when they become old. Table 4.22 contains data on this measure. From the table it is found that in old

(C) The International Journal of Indian Psychology, ISSN 2348-5396 (e)| ISSN: 2349-3429 (p) | 145 


\section{Social Construction of Aging}

age the support that they most expect from their children and family members is emotional support (86.33\%). As compared to females this percentage is higher among males (91.33\%). Next important support that got mentioned was physical support (77.66\%).

\begin{tabular}{|c|c|c|c|c|c|c|c|c|c|c|}
\hline & & & Males & & & & Females & & & \\
\hline & & 1 & 2 & 3 & 4 & 5 & 6 & 7 & 8 & 9 \\
\hline 'Z & 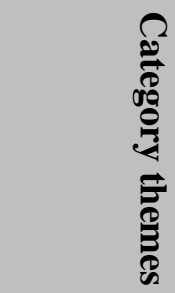 & 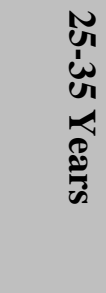 & 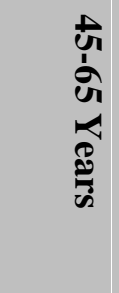 & 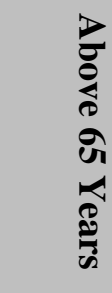 & 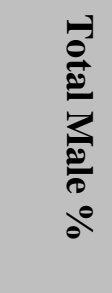 & 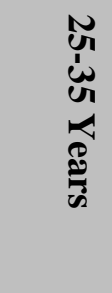 & 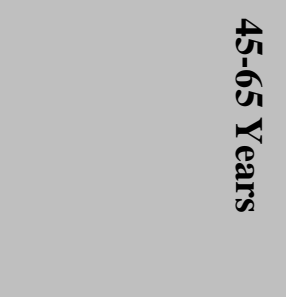 & 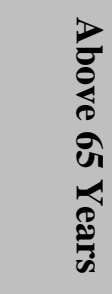 & 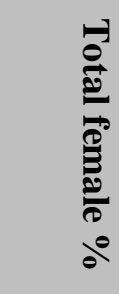 & 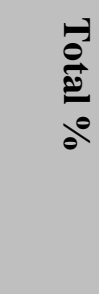 \\
\hline 1 & Financial & 70.00 & 74.00 & 80.00 & 74.66 & 88.00 & 64.00 & 72.00 & 74.66 & 74.66 \\
\hline 2 & Societal & 40.00 & 38.00 & 40.00 & 39.33 & 56.00 & 68.00 & 58.00 & 68.66 & 50.00 \\
\hline 3 & Emotional & 88.00 & 90.00 & 96.00 & 91.33 & 74.00 & 84.00 & 86.00 & 81.33 & 86.33 \\
\hline 4 & Physical & 64.00 & 90.00 & 92.00 & 82.00 & 70.00 & 74.00 & 96.00 & 80.00 & 77.66 \\
\hline
\end{tabular}

\section{(vii) Receiving advice from parents}

It contains data about the extent to which adult respondents of today would welcome advice from their old parents in future. For this a four point rating scale was developed which ranged from 1'not at all' to 4 - 'too much'. The data analysis indicated that the total sample inclusive of all males and females said that they would accept only "a bit" of advice from their old parents in future.

\begin{tabular}{|c|c|c|c|c|c|c|c|c|c|c|}
\hline & & & Males & & & & Females & & & \\
\hline & & 1 & 2 & 3 & 4 & 5 & 6 & 7 & 8 & 9 \\
\hline z & 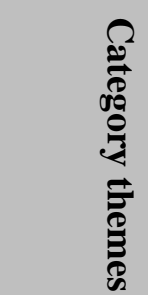 & 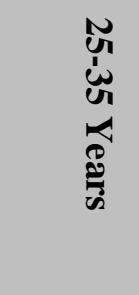 & 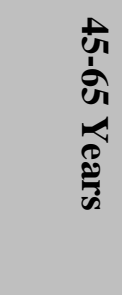 & 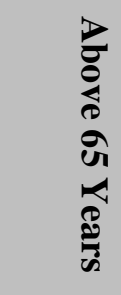 & 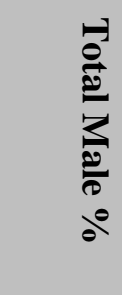 & 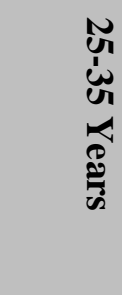 & 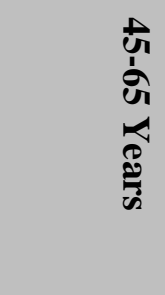 & 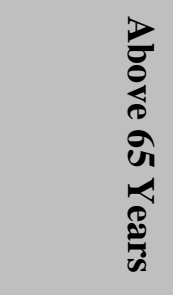 & 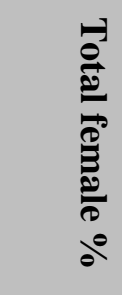 & $\frac{\overrightarrow{0}}{0^{\circ}}$ \\
\hline 1 & $\begin{array}{l}\text { Not at } \\
\text { all }\end{array}$ & & & & & & & & & \\
\hline 2 & $\begin{array}{l}\text { Little } \\
\text { bit }\end{array}$ & & & & & & & & & \\
\hline 3 & A bit & 100.00 & 100.00 & 100.00 & 100.00 & 100.00 & 100.00 & 100.00 & 100.00 & $\begin{array}{l}100.0 \\
0\end{array}$ \\
\hline 4 & $\begin{array}{l}\text { Too } \\
\text { much }\end{array}$ & - & - & - & - & - & - & - & - & - \\
\hline
\end{tabular}

(C) The International Journal of Indian Psychology, ISSN 2348-5396 (e)| ISSN: 2349-3429 (p) | 146 


\section{Social Construction of Aging}

(viii) Relationship with old parents

For this a four point rating scale was developed ranging from $1=$ Not close at all, 2 = A little close, 3 = somewhat close and $4=$ very close. The data analysis indicated that the total sample said that they would be "very close" to their old parents in future.

\begin{tabular}{|c|c|c|c|c|c|c|c|c|c|c|}
\hline & & & Males & & & & Females & & & \\
\hline & & 1 & 2 & 3 & 4 & 5 & 6 & 7 & 8 & 9 \\
\hline zu & 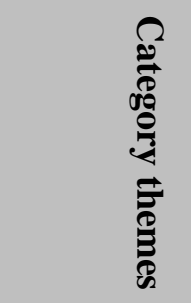 & 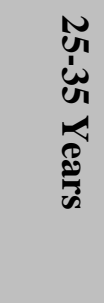 & 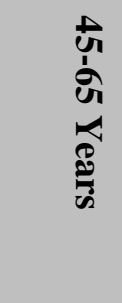 & 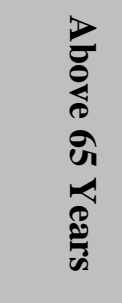 & 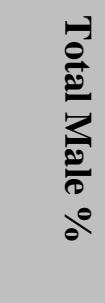 & 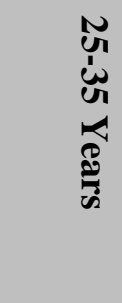 & 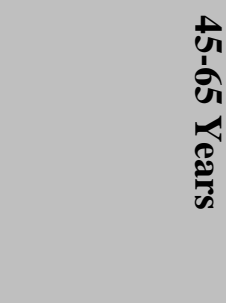 & 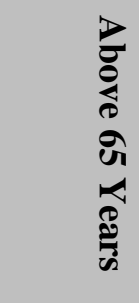 & 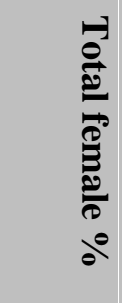 & 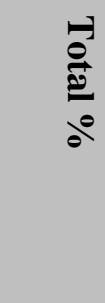 \\
\hline 1 & $\begin{array}{l}\text { Not close } \\
\text { at all }\end{array}$ & & & & & & & & & \\
\hline 2 & $\begin{array}{l}\text { A little } \\
\text { close }\end{array}$ & - & - & - & - & - & - & - & - & - \\
\hline 3 & $\begin{array}{l}\text { Somewhat } \\
\text { close }\end{array}$ & 4.00 & 10.00 & 4.00 & 6.00 & - & - & - & - & - \\
\hline 4 & $\begin{array}{l}\text { Very } \\
\text { close }\end{array}$ & 96.00 & 90.00 & 96.00 & 94.00 & 100.00 & 100.00 & 100.00 & 100.00 & 97.00 \\
\hline
\end{tabular}

\section{DISCUSSION}

In the present study the issue of age as a cause of social inequality is being analyzed from the social constructivist perspective. The adoption of this perception stem from conviction that age has ceased to be a purely biological or natural categorization and with the beginning of modern times and the changes in the functioning of societies it fluctuated into being a socially and culturally constructed phenomenon.

The ways the younger generations conceive of old age is rather valuable data firstly because these generations will become old in the future. Hence, in order to make projections about how old age phenomenon will look like after about 50 years, it is efficacious to take the considerations of younger people seriously, especially middle aged people, because young people none of whom had no way to have already experienced the old are before, are too far to the idea of being old.

Aging concerns the middle- aged more than other age groups as they see their future in old people. The middle- aged together with the elderly can make the attitudes and opinions about aging in society more positive. People construct all kind of knowledge about the social world both through getting involved in it and being the other for it. In construction of an object of

(c) The International Journal of Indian Psychology, ISSN 2348-5396 (e)| ISSN: 2349-3429 (p) | 147 


\section{Social Construction of Aging}

knowledge, otherness is more powerful, yet based more on perception. Therefore, young people construct old age not by experiencing it; however as another within this phenomenon, they produce this knowledge through their perceptions. They perceive what has been produced, and then reproduce it. As a matter of fact, according to Haim Hazan (1994) a study of socially constructed old age consists of two parts: handling old age as a socio-cultural object among the non-olds and how old people construct their own world.

The questions in questionnaire are organized in order to grasp the constructed realities about aging in general and ageing in particular. Moreover, it is also significant to keep in mind that people construct their own identities as the other see us. When the questions "worries after 20-30 years" has been asked then most of the respondents asked that "poor health" is the main worry after 20-30 years. The main reason for this difference is because of the stereotypic beliefs about the elderly person which are widespread in our society. As Nelson (2002) states "age prejudice is one of the most condoned, institutionalized form of prejudice in the world today".

When the question "who is responsible for caring for older people" was asked, the respondents in the younger, middle as well as the older generation reported that children are the major source who are responsible for caring for their older parents. Similarly, the question of whether 'Adult's responsibility is more towards their own children or else their old parents' $100 \%$ of the respondents reported that they are equally responsible for their children as well as their parents. Thus, at least, at the cognitive level, younger generation also is found to verbalize their responsibilities towards their older parents. Filial responsibility is conceptualized as a societal attitude towards the duty of adult children to meet the needs of their aging parents (Seelbach, 1981; Walker et. al., 1990). These attitudes relate to the felt duties of adult children and to the attitudes of elderly individuals regarding the obligations of young family members to care and protect their elderly parents (Hanson, Seelbach and Seelbach, 1983). Filial obligation to elderly parents and their care is a very important religious and moral commandment in India, perhaps much more than in other societies, and includes provision of material and financial assistance as well as instrumental help (Linzer, 1986 \& Sapp, 1996). The young adults know that they have to take care of their children and help them enter to live comfortable lives even in old age. However, at the same time, they are also aware that assisting their parents in old age would be difficult because of simultaneous demands from their wives and children and their preference for being independent and away. They expressed that their parents are the greatest strength for them. In this paper the attitudes toward the conception of successful aging were studied with different statements. Statements "how would you make yourself useful after 20-30 years from now", was highly positively evaluated by the respondents. When the question "what will be the possibility of getting support at that age" was asked to the respondents then all the respondents of different age groups reported that they get $80-90 \%$ of support by their children when they become old. Although most of the respondents of different age groups, either male or female, reported that every day they spend their time with their older parents when statement "How much time would 


\section{Social Construction of Aging}

you spend with your old parents after 20-30 years from now" was asked to them. For the statement like "Relationship you would have with your parents" was asked most respondents said that they would be very close to their parents after 20-30 years from now.

These statements are highly positively evaluated by the respondents. Individuality is the thing to be appreciated. The attitude of society towards aging and old age reflects the maturity and level of ethics of society. All people especially elderly and middle aged have the task to improve the reputation of aging and take favorable stance towards their own healthy and successful aging. For construing old age in a positive manner, the middle-age is the ripe time. The middle aged know that preparation for aging should start early, but most have made no conscious efforts for that.

"Today the old and young, members of different historical cohorts, know less and less about each other and share little of each other's cultural worlds" (Moody, 1993). In this paper it is mainly aimed to assess the degree to which young people and old people have an idea about old people's world. In addition, young people contribute to social construction of old age phenomenon by participating to his construction as other; but they inherently know that they are potentially old. Differentiation between young and old is perhaps the only one in which people of privileged side know that they will turn out to be unprivileged on day. Moreover, how younger and older people perceive old age and ageing process is of great importance. "How humans conceive of their worlds affects profoundly how they anticipate and create their future" (Atchely, 1993 : 4). Future population of older people will be more culturally and socially diverse than past cohorts of older people, as well as better educated and in better health, more inclined to maintain active lifestyles, and less inclined to accept uniform and stereotypical notions of what it means to be old" (Polivka, 2009: 562). The anticipations about elderly in future are so crucial for developing social policies in regard to meet the needs of old people.

\section{Acknowledgments}

The author appreciates all those who participated in the study and helped to facilitate the research process.

\section{Conflict of Interests}

The author declared no conflict of interests.

\section{REFERENCE}

Atchely, R. C., (1993) critical perspectives on retirement. In T.R. cole. W. A. Achenbaum, P.L. Jakobi, \& R. kastenbaum (Eds.). voices and visions of aging: toward a critical gerontology (pp. 3-19). New York: springer publishing.

Berger, P.L. and T. Luckmann (1966). The Social Construction of Reality: A Treatise in the sociology of knowledge, Garden City, NY: Anchor Books. ISBN O-385-05898-5. 


\section{Social Construction of Aging}

Craig, R.T. (1995). Foewword. In E. Leeds - Hurwitz (Ed.), Social approaches to communication (PP. v-ix). New York: Guilford Press.

Hanson, S.L., Saur, W.J. and Seelbach, W.C., 1983, Racial and Cohort variations in filial responsibility norms. Gerontologist 236, pp. 626-631.

Hareven, T. K., 2005, "Changing Images of Aging and the Social Construction of the Life Course", Featherstone, M. And Wernick A. (ed.) Images of Aging: Cultural Representations of Later Life, Routledge: London

Hazan H., 1994, Old Age: Constructions and Deconstructions, Cambridge University Press: Cambridge.

Linzer, N. 1986. The obligation of adult children to aged persons: a view from Jewish tradition. Journal of Aging and Judaism 11, pp. 34-38.

Moody, H.,1993, “ Overview: What is Critical Gerontology and Why Is It Important?”, Cole, T. R. et al (ed), Voices and Visions of Aging: Toward a Critical Gerontology, Springer Publishing Company: New York

Nelson, T.D. (2002). Preface. In T.D. Nelson (Ed.), Ageism: Stereotyping and Prejudice against older adults. Cambridge, MA: MIT Press.

Polivka L., 2009, “Gerontology for the 21st Century”. The Gerontologist, Aug 2006; 46:4, pp.558-563.

Seelbach, W.C., 1981. Filial responsibility among aged parents: a racial comparison. Journal of Minority Aging 5, pp. 286-292.

Walker, A.J., Pratt, C.C., Shinn, H. and Jones, L.L. (1990). Motives for Parental Caregiving and Relationship quality. Family Relations, 39, pp. 51-56.

How to cite this article: R Saxena (2016), Social Construction of Aging, International Journal of Indian Psychology, Volume 3, Issue 4, No. 64, ISSN 2348-5396 (e), ISSN: 2349-3429 (p), DIP: 18.01.129/20160304, ISBN: 978-1-365-32519-9 